\title{
Designing the Optimal Quantitative Electron Probe X-ray Microanalysis Measurement
}

\author{
Nicholas W. M. Ritchie, Dale E. Newbury \\ National Institute of Standards and Technology, Materials Measurement Science Division, \\ Gaithersburg, MD 20899
}

Designing the optimal electron probe x-ray microanalysis measurement is difficult for both novice and expert. There are a relatively small number of parameters to be optimized but beyond some rules-ofthumb, there is little concrete guidance on how to perform the optimization. For example, the optimization depends on selecting a beam energy, the optimal choice of which is dependent upon choice of standard material and x-ray transition (K $\alpha$ vs. L $\alpha$ vs. M $\alpha$ etc.) Rules-of-thumb and experience allow experts to select a reasonable transition and standard[1]. Furthermore, for EDS measurements one must also consider which reference spectra are required. Standard spectra provide x-ray intensity information and reference spectra provide pure spectral lineshape information without interferences from other elements. The choices of reference spectra are largely dependent upon the choice of standard. Simple standards often don't require separate references but typically produce less accurate measurements than multi-element standards of similar composition to the unknown. However, it can be a challenge even for the experienced microanalyst to determine which references are required since the choice depends upon details of the implementation of the spectrum fitting algorithm. Furthermore, one must make a trade-off between precision and patience. The net result is that in practice performing standards-based quantification is often viewed as daunting and as a result many measurements which require the accuracy and precision of standards-based analysis are performed using less reliable standard-less analysis.

To assist both the novice and expert, we have designed the first of its kind tool for optimizing microanalytical measurement protocols. This tool is based on the algorithms presented by Ritchie and Newbury[2]. This paper describes how to calculate the effect of uncertainty in the mass absorption coefficient and in the backscatter coefficient on the matrix correction. This paper represents the first time that it is possible to make estimates of the accuracy of a microanalysis measurement that goes beyond accounting for measurement reproducibility.

In general, the optimal measurement is the one most suited for the purpose but, in practice, the optimal measurement is the most accurate measurement given a realistic set of constraints. With our new ability to estimate the accuracy of a measurement protocol, it is possible to numerically compare various different experimental schemes to determine the one that best satisfies the requirements. Constraints might include time, instrument and detector performance, the availability of standard materials and the accuracy with which the composition of the standards is known. The input to such an optimization algorithm is an estimate of the composition of the unknown. This estimate may be the result of a previous sub-optimal measurement - either performed with standards or with standard-less analysis.

The algorithm performs a grid search in the experimental design space. At each experimental configuration (beam energy, choice of standards, etc.), the algorithm calculates an estimate of the measurement accuracy. The algorithm then assigns a metric which allows the operator to compare alternatives and proposes an experimental design which minimizes the total uncertainty budget. Alternatively, a user might want to optimize the accuracy with which a particular element is measured. 
The result of the optimization is presented as a recipe which details the instrument parameters and the spectra which need to be collected. The report also suggests the minimal required set of standard blocks based on the materials required and a user-entered database of standard materials and multi-standard blocks.

[1] J. Goldstein, D. Newbury, D. Joy, C, Lyman, P. Echlin, E. Lifshin, L. Sawyer, and J. Michael., "Scanning Electron Microscopy and X-Ray Microanalysis", Kluwer Academic, Plenum Publishers, New York; 2003

[2] N. Ritchie and D. Newbury, Anal. Chem, Anal. Chem. 2012, 84, 9956-9962

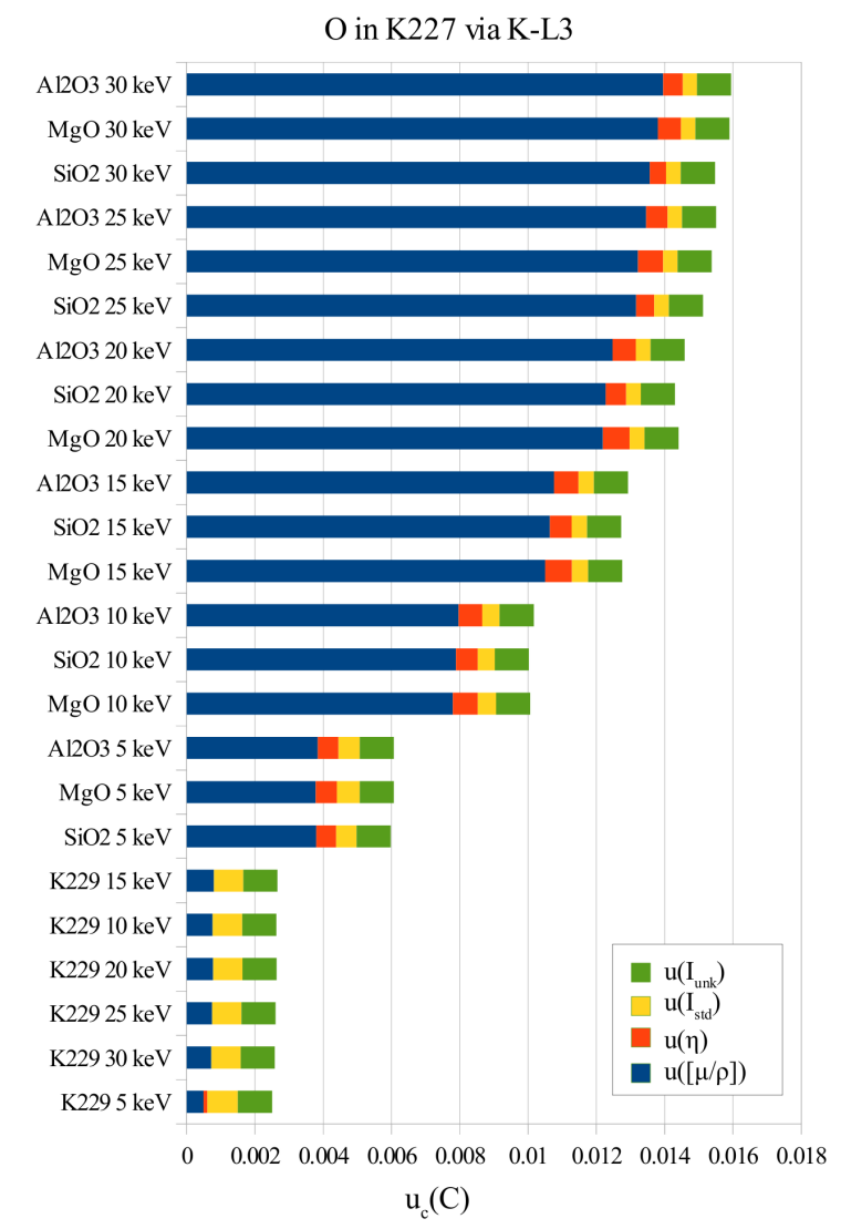

Figure 1: The uncertainty budget for measuring oxygen in NIST glass K227 ( $\mathrm{Si}, \mathrm{Pb}$ and $\mathrm{O}$ ) depends upon both the choice of standard material and the beam energy. Four different components of the error budget are summarized by color in this plot. Green (unknown) and yellow (standard) represent the contribution of count statistics, red represents the contribution of uncertainty in the backscatter coefficient and blue represents the contribution of uncertainty in the mass absorption coefficient. 